\title{
14-3-3 $\varepsilon$ protein increases matrix metalloproteinase-2 gene expression via p38 MAPK signaling in NIH3T3 fibroblast cells
}

\author{
Eun Kyung Lee ${ }^{1,2 *}$, Youn Sook Lee ${ }^{1 *}$, Hansol Lee ${ }^{3}$, \\ Cheol Yong Choi ${ }^{1}$ and Seok Hee Park ${ }^{1,4}$
}

\author{
${ }^{1}$ Department of Biological Science \\ Sungkyunkwan University \\ Suwon 440-746, Korea \\ ${ }^{2}$ Inha University College of Medicine \\ Incheon 400-121, Korea \\ ${ }^{3}$ Department of Biological Sciences \\ Inha University \\ Incheon 402-751, Korea \\ ${ }^{4}$ Corresponding author: Tel, 82-31-290-5912; \\ Fax, 82-31-290-7015; E-mail, parks@skku.edu \\ *These authors contributed equally to this work. \\ DOI 10.3858/emm.2009.41.7.050
}

Accepted 30 January 2009

Abbreviations: DN-p38 MAPK, dominant negative p38 MAPK; DN-SEK, dominant negative stress-activated protein kinase; ECM, extracellular matrix; MMP, matrix metalloproteinase; PI3-kinase, phosphatidylinositide 3-kinase

\footnotetext{
Abstract

One of the 14-3-3 protein isoforms, $14-3-3 \varepsilon$, was previously shown to be increased during skin aging. We suggest here a possible role for the 14-3-3 $\varepsilon$ protein in skin aging by providing evidence that $14-3-3 \varepsilon$ increases the expression of the matrix-metalloproteinase (MMP)-2 gene in NIH3T3 fibroblast cells. Expression of the 14-3-3 $\varepsilon$ gene in NIH3T3 cells primarily up-regulated the expression of the MMP-2 gene at the transcriptional level by inducing specific DNA binding proteins bound to an upstream region of the MMP-2 promoter from $-1,629$ to $-1,612$. Inhibition of endogenous $14-3-3 \varepsilon$ gene expression by RNA interference also decreased endogenous MMP-2 gene expression. Furthermore, up-regulation of the MMP-2 gene by 14-3-3 $\varepsilon$ was suppressed by expression of a dominant-negative mutant of p38 MAP kinase. These findings strongly suggest that increased expression of $14-3-3 \varepsilon$ contributes to remodeling of extracellular matrix in skin through increasing MMP-2 gene expression via p38 MAP kinase signaling.
}

Keywords: $14-3-3$ proteins; extracellular matrix; matrix metalloproteinase 2; p38 mitogen-activated protein kinases; signal transduction; skin aging

\section{Introduction}

Matrix metalloproteinases (MMPs) are a large family of zinc-dependent endopeptidases that collectively degrade several extracellular matrix (ECM) constituents. They are subdivided into six different classes based on their substrate specificities, sequence similarities and domain organization: collagenases, gelatinases, stromelysins, matrilysins, membranetype MMPs and others (Visse and Nagase, 2003). The activities of most MMPs are low in normal tissues at steady-state. However, their expressions can be transcriptionally induced by a variety of growth factors, inflammatory cytokines, hormones, cell-cell and cell-matrix interactions (Nagase and Woessner, 1999). Because MMPs are primarily responsible for ECM turnover and connective tissue remodeling, accumulating evidence indicates that an imbalance in the expressions of MMPs under pathological conditions are related to human diseases, including dermal fibrosis, skin aging and cancer (Fisher et al., 1996; Ghahary et al., 1996; Nagase and Woessner, 1999; Brinckerhoff and Matrisian, 2002; Rittie and Fisher, 2002).

Among these pathological phenomena, marked degradation and degenerative changes of ECM in the dermis were recognized to give rise to wrinkle formation during the skin aging process, and thus the increased expression of MMPs was assumed to be involved in wrinkle formation in skin (Fisher et al., 1996, 1997; Varani et al., 2000; Rittie and Fisher, 2002). Among the MMPs, it was recently reported that the activation of gelatinases, including MMP-2 and MMP-9, played important roles in ultraviolet-induced skin aging in hairless mice (Inomata et al., 2003) and MMP-2 was expressed in human dermal fibroblasts (Scharffetter et al., 1991).

The 14-3-3 proteins constitute a family of highly conserved acidic proteins expressed in wide range of organisms and tissues (Fu et al., 2000; van Hemert et al., 2001; Wilker and Yaffe, 2004). There are seven mammalian isoforms of the 14-3-3 family $(\beta, \varepsilon, \eta, \gamma, \theta, \xi$ and $\sigma$ ) that are related to a variety of cellular functions, including the regulation 
of cell signaling, cell cycle progression, apoptosis, stress responses, cytoskeleton organization, cell migration and malignant transformation (Fu et al., 2000; van Hemert et al., 2001; Wilker and Yaffe,
2004). Of the seven 14-3-3 protein isoforms, $14-3-3 \varepsilon$ has been implicated in neuronal development, cell migration, human topoisomerase Il $\alpha$ function and skin aging (Kurz et al., 2000; Toyo-oka et al., 2003;
A

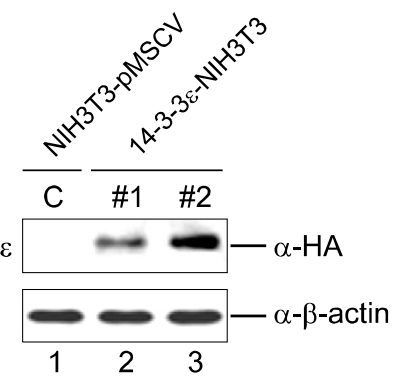

B

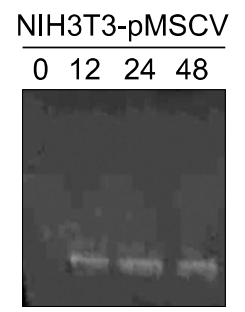

14-3-3e-NIH3T3
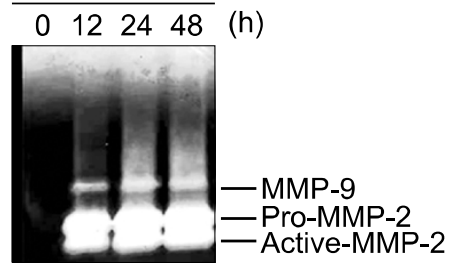

C

\author{
NIH3T3-pMSCV $14-3-3 \varepsilon-N I H 3 T 3$$$
\begin{array}{lllllllll}
\hline 0 & 12 & 24 & 48 & & 0 & 12 & 24 & 48
\end{array} \text { (h) }
$$ \\ MMP-2 \\ MMP-9 \\ $\beta$-actin
}

D

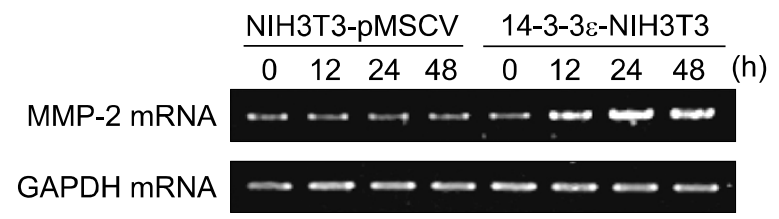

E

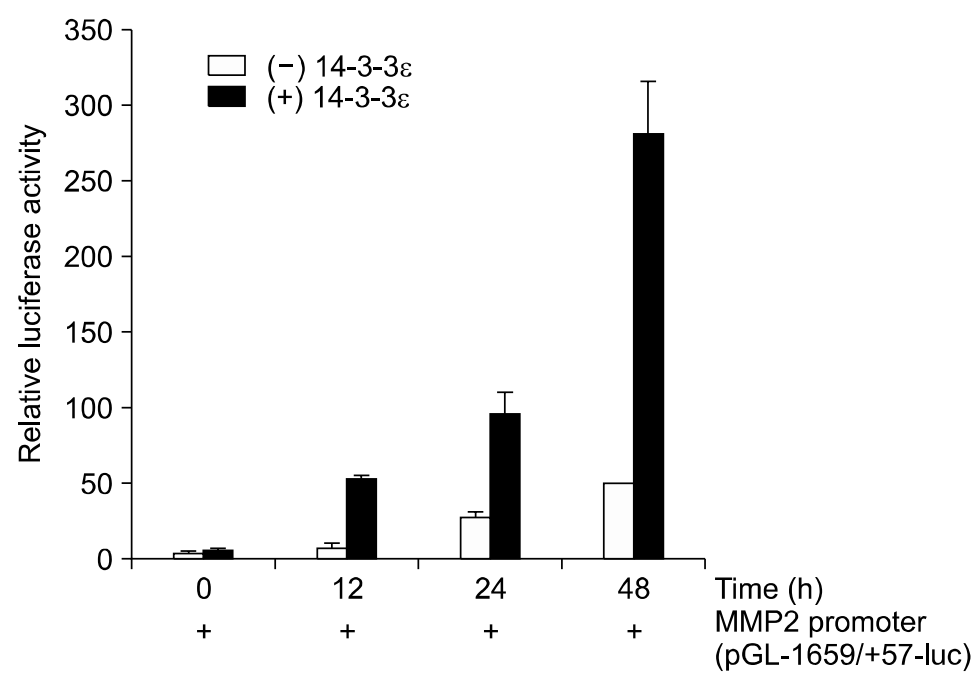

Figure 1. Protein $14-3-3 \varepsilon$ increases MMP-2 gene expression in NIH3T3 fibroblast cells. (A) Construction of stable cell lines expressing the $14-3-3 \varepsilon$ gene. Cell lysates of NIH3T3 cells, either stably expressing the 14-3-3 $\varepsilon$ gene tagged with HA epitope (lane 2 and 3 ) or empty pMSCV-puro vector (lane 1), were immunoblotted with anti-HA antibody. Gelatinolytic activities of MMP-2 and MMP-9 proteins and their expressions were analyzed in 14-3-3ع- expressing and pMSCV-puroexpressing NIH3T3 cells by gelatin zymogram assay (B) and Western blot analysis (C). Conditioned media for gelatin zymography and cell lysates were obtained at the indicated times. (D) Expressions of MMP-2 mRNA were analyzed by RT-PCR analysis in control NIH3T3 cells and 14-3-3e-expressing cells. (E) NIH3T3 cells were transiently transfected with the full-length MMP-2 promoter (pGL-1659/+57-luc) in the presence or absence of the 14-3-3 $\varepsilon$ gene. Cells were subsequently incubated for the indicated times, and luciferase activities were normalized on the basis of $\beta$-galactosidase expression to adjust for variations in transfection efficiency. Results are mean values $\pm \mathrm{SD}$ of three independent experiments. 
Choi et al., 2005; Tak et al., 2007).

We previously demonstrated that $14-3-3 \varepsilon$ was highly expressed in intrinsically aged and photoaged human skin (Choi et al., 2005). However, it remains unknown how increased expression of 14-3-3e contributes to the process of skin aging. In this study, we hypothesized that $14-3-3 \varepsilon$ affects ECM turnover and examined the expressions of MMP genes in NIH3T3 fibroblast cells. We propose here that $14-3-3 \varepsilon$ can increase the expression of the MMP-2 gene by induction of specific DNA binding proteins bound to a distinct region from $-1,629$ to $-1,612$ of the MMP-2 promoter via p38 MAPK signaling, which eventually leads to alterations of ECM components.

\section{Results}

Cumulative degradations and degenerative changes of extracellular matrix (ECM) in dermis are important characteristics of skin aging phenotypes, causing wrinkle formation, and the increased expressions of MMPs are thought to be related to ECM degradations (Fisher et al., 1996, 1997; Varani et al., 2000; Rittie and Fisher, 2002). We previously reported that the $14-3-3 \varepsilon$ protein isoform was increased in intrinsically aged and photoaged human skin (Choi et al., 2005), although the reason was not identified. We thus hypothesized that the increased expression of $14-3-3 \varepsilon$ may affect ECM turnover. In order to verify our hypothesis, we initially constructed stable cells expressing the 14-3-3e gene in NIH3T3 fibroblast cells. Retroviruses expressing the $14-3-3 \varepsilon$ gene, tagged with an HA epitope, were infected into NIH3T3 cells, and puromycin-resistant cells were isolated. Immunoblot analysis showed that the $14-3-3 \varepsilon$ gene was highly expressed in puromycin-resistant cells and a clone, designated \# 2, was used for further experiments (Figure 1A).

Using this stable cell line expressing 14-3-3e, we first examined the expressions of the gelatinases MMP-2 and MMP-9. These degrade ECM proteins, such as type IV collagen, elastin, vitronectin and aggrecan, and MMP-2 activity is highly expressed in intrinsically aged and photoaged human skin (Chung et al., 2001). As shown by gelatinase zymography using conditioned media obtained from 14-3-3e-expressing NIH3T3 cells at the indicated times post-plating, MMP-2 activity was dramatically increased in 14-3-3e-expressing cells compared with control cells expressing an empty vector (Figure 1B). Gelatinase activity for MMP-9 was also augmented in 14-3-3e-expressing cells, although its activity was less than that for MMP-2 (Figure
1B).

Using cell lysates from 14-3-3e-expressing cells and control cells, immunoblot analysis revealed that $14-3-3 \varepsilon$ increased the expression of MMP-2 protein, and stimulated the production of active MMP-2 from pro-MMP-2 at the indicated times post-plating (Figure 1C). In contrast, the expression of MMP-9 protein was not significantly affected by the $14-3-3 \varepsilon$ protein, although the reasons remain unknown (Figure 1C).

Next, we examined if the increased expression of MMP-2 protein by $14-3-3 \varepsilon$ was due to the induction of MMP-2 mRNA. RT-PCR analysis in 14-3-3e-expressing cells and control cells showed that $14-3-3 \varepsilon$ up-regulated the expression of MMP-2 mRNA (Figure 1D). These results were also confirmed by the induction of full-length MMP-2 promoter activity by $14-3-3 \varepsilon$ (Figure $1 \mathrm{E}$ ). These findings strongly suggest that $14-3-3 \varepsilon$ primarily increases the expression of the MMP-2 gene at the tran-

A

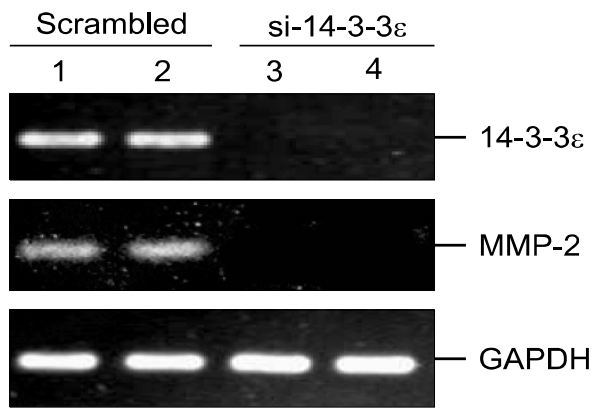

B

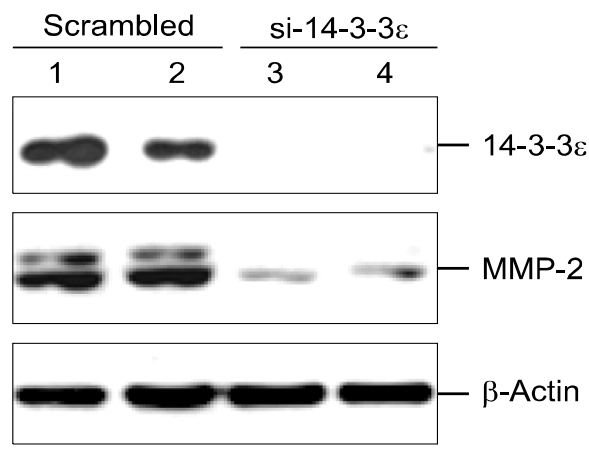

Figure 2. Endogenous inhibition of the 14-3-3 $\varepsilon$ gene using 14-3-3 $\varepsilon$-specific siRNA decreases the expression of the MMP-2 gene in NIH3T3 cells. Cells were independently transfected with either scrambled siRNA

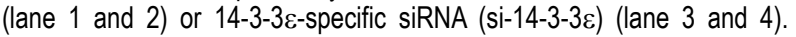
Cells were incubated for $24 \mathrm{~h}$ and RT-PCR $(\mathrm{A})$ and Western blot $(\mathrm{B})$ analyses were performed. 
scriptional level, and also affects the production of active MMP-2 protein. Therefore, these results prompted us to focus on the mechanism for the induction of the MMP-2 gene by $14-3-3 \varepsilon$.

To clearly verify a role for $14-3-3 \varepsilon$ in the increased expression of the MMP-2 gene, NIH3T3
A

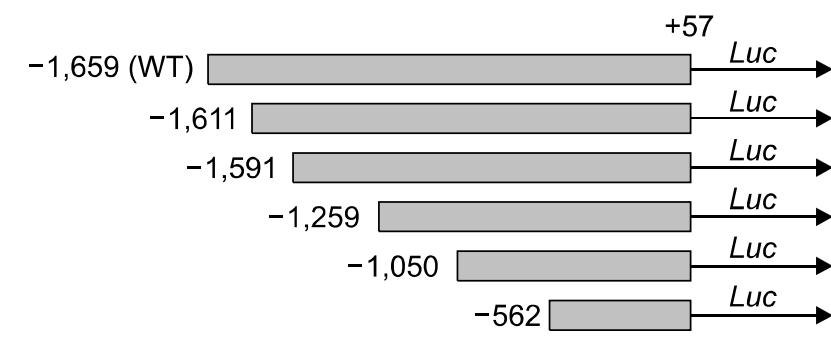

C

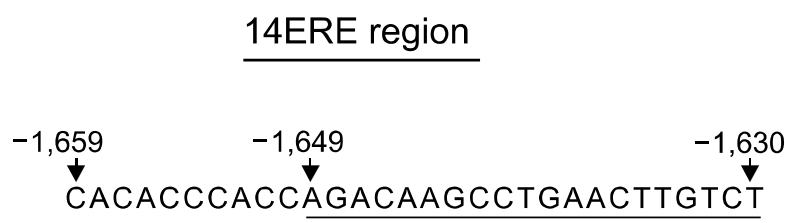
p53BS

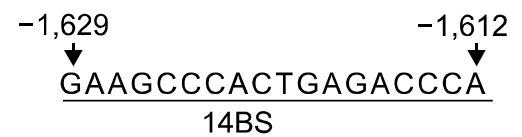

$\mathbf{F}$

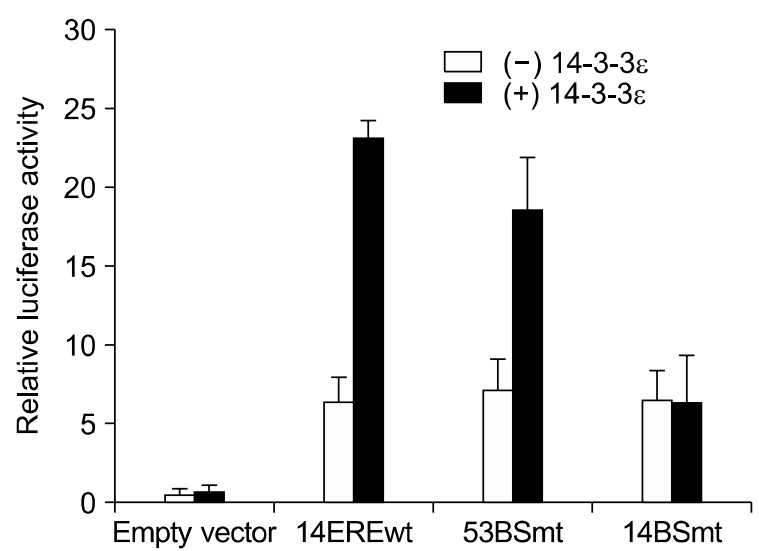

B

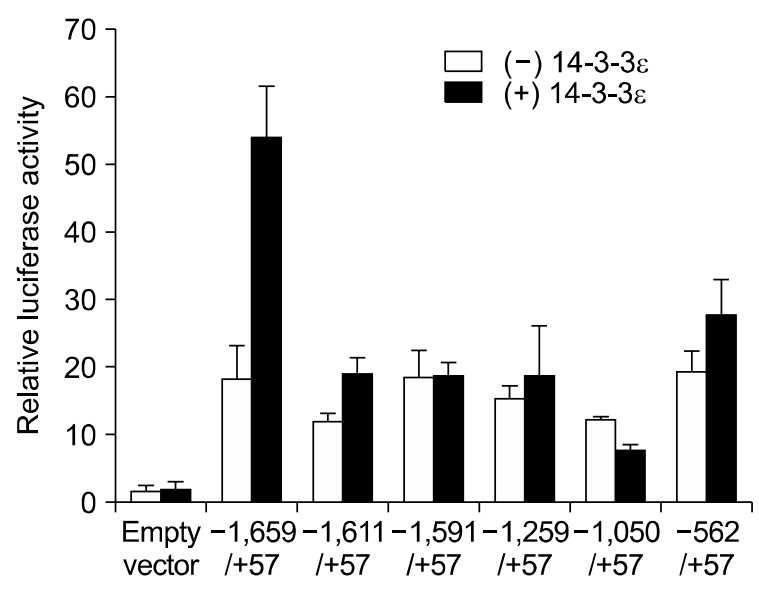

D

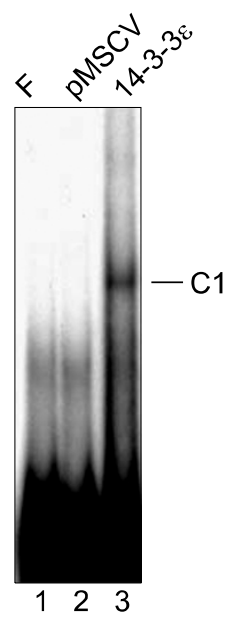

E

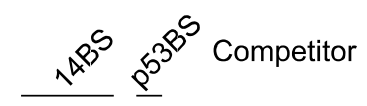

F $0105050 \times$ Molar ratios

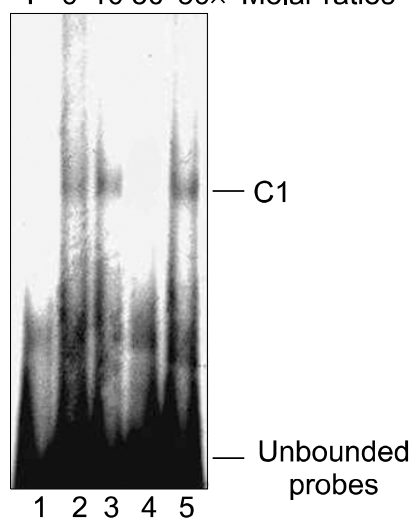

Figure 3. Protein 14-3-3 $\varepsilon$ activates the MMP-2 promoter by increasing specific DNA binding proteins bound to the region -1,629 to -1,612 (14BS sequences). (A) Schematic representations of serial deletion mutants of the MMP-2 promoter. (B) Serial deletion mutants of the MMP-2 promoter were transiently transfected into NIH3T3 cells in the presence or absence of the 14-3-3 $\varepsilon$ gene, and luciferase activities were analyzed. The luciferase activities were normalized on the basis of $\beta$-galactosidase expression to adjust for variations in transfection efficiency. All results are mean values $\pm S D$ of three independent experiments. (C) Sequences of the 14-3-3ع-response element (14ERE). The regions from -1,649 to -1,630 and from -1,629 to $-1,612$ are designated as p53BS and 14BS, respectively. (D) Electrophoretic mobility shift assays (EMSA) were performed with labeled double-stranded oligonucleotides containing the 14ERE region and nuclear extracts of either pMSCV-puro-expressing (lane 2) or 14-3-3e-expressing (lane 3) NIH3T3 cells. Lane 1: free probe. (E) Competition assays were performed with labeled 14ERE oligonucleotides and nuclear extracts of 14-3-3e-expressing NIH3T3 cells according to the indicated molar ratios of cold competitors. Unlabeled double-stranded oligonucleotides with 14BS sequences $(-1,629$ to $-1,612)$ and p53BS $(-1,649$ to $-1,630$ ) were used as competitors. Lane 1; free probe, Lane 2; no competitor. $(F)$ To verify if the 14BS region of the MMP-2 promoter plays an important role in 14-3-3e-mediated MMP-2 expression, NIH3T3 cells were transfected with p14EREwt-luc (wild type), p53BSmt-luc (mutation in p53BS region) and p14BSmt-luc (mutations in 14BS region) plasmids in the presence or absence of $14-3-3 \varepsilon$ gene, respectively. 
cells were treated with either siRNA for the 14-3-3e gene or scrambled SiRNA. As shown by RT-PCR and immunoblot analyses, treatment with $14-3-3 \varepsilon-$ specific siRNA profoundly decreased the expressions of endogenous 14-3-3e mRNA and protein and subsequently caused the reductions of endogenous MMP-2 mRNA and protein (Figure 2A and $B)$. These results strongly support our finding that the expression of the $14-3-3 \varepsilon$ gene up-regulates the expression of the MMP-2 gene in NIH3T3 fibroblast cells.

We next investigated how $14-3-3 \varepsilon$ increased the expression of MMP-2 mRNA at the transcriptional level. To identify the underlying mechanism, we constructed serial deletion mutants of the MMP-2 promoter linked to a reporter gene (Figure $3 A$ ). These mutant plasmids were co-transfected into NIH3T3 cells in the presence or absence of a 14-3-3e expression plasmid. As shown in Figure $3 \mathrm{~B}$, the full length promoter of MMP-2 $(-1,659 /+57)$ was significantly induced by $14-3-3 \varepsilon$. However, when the region from $-1,659$ to $-1,612$ was deleted, the induction of MMP-2 promoter by $14-3-3 \varepsilon$ was profoundly reduced compared with other deletion mutants (Figure $3 \mathrm{~B}$ ). These results indicate that the region from $-1,659$ to $-1,612$, designated as

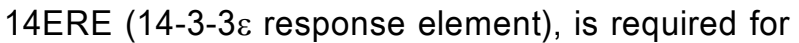
the induction of the MMP-2 promoter by $14-3-3 \varepsilon$ (Figure 3C).

As members of the 14-3-3 protein family do not directly bind to specific DNA sequences, we examined if $14-3-3 \varepsilon$ expression might induce the specific DNA binding proteins bound to the 14ERE region of the MMP-2 promoter. Double-stranded oligonucleotides with 14ERE sequences were labeled with ${ }^{32} \mathrm{P}$, and EMSA was performed with nuclear extracts from 14-3-3e-expressing and control NIH3T3 cells. As shown in Figure 3D, expression of the 14-3-3 $\varepsilon$ protein induced a distinct DNA-protein complex, denoted C1. To confirm if this DNA-protein complex was specific for 14ERE, competition assay was performed. Interestingly, because it is known that the 14ERE region from $-1,659$ to $-1,612$ of the MMP-2 promoter contains the binding site $(-1,649$ to $-1,630$ ) for the p53 protein (Qin et al., 1999), we designed two double-stranded oligonucleotides, designated p53BS and 14BS (Figure 3C), and used their unlabelled oligonucleotides as cold competitors for competition assay. As shown in Figure 3E, the DNA-protein complex, denoted $\mathrm{C} 1$, was in specific competition with the 14BS oligonucleotide, but not with p53BS (Figure 3D). These results indicate that $14-3-3 \varepsilon$ expression significantly induces a specific DNA binding protein that binds to the 14BS sequence, which contributes to inducing the activation of the MMP-2 promoter.

Considering our present results, we next asked if the 14BS sequence of the MMP-2 promoter acted as an important cis-acting element for 14-3-3عmediated MMP-2 expression. To address this que-
A

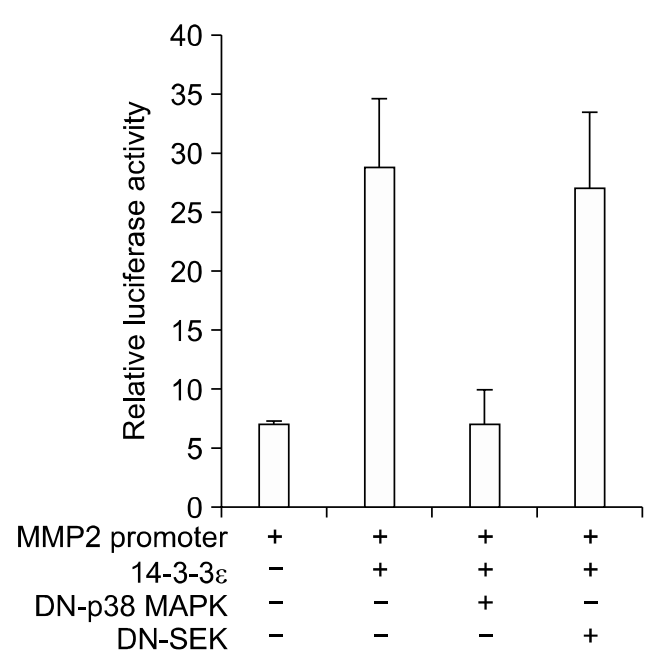

B

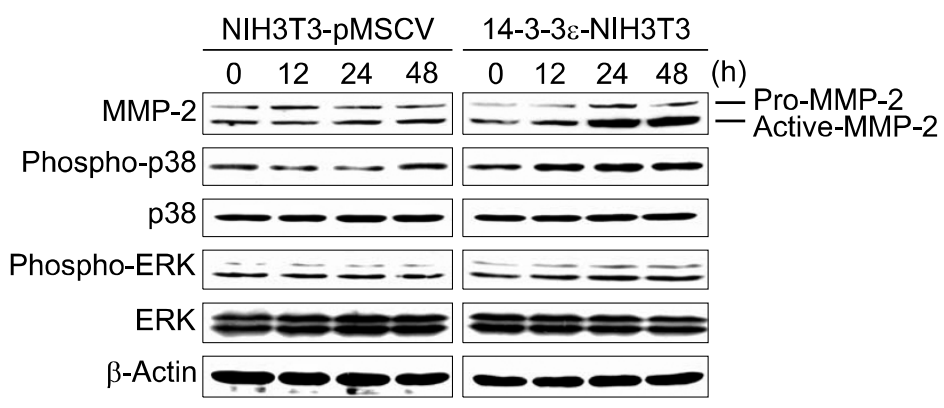

Figure 4. 14-3-3 $\varepsilon$ activates the MMP-2 promoter via p38 MAPK signaling. (A) The full-length MMP-2 promoter-luciferase reporter (pGL-1,659/+57-luc) and 14-3-3 $\varepsilon$ expression plasmid were co-transfected into NIH3T3 cells together with a plasmid expressing either a dominant negative p38 MAPK (DN-p38 MAPK) or a dominant negative SEK (DN-SEK). The luciferase activities were normalized on the basis of $\beta$-galactosidase expression to adjust for variations in transfection efficiency. All results are mean values \pm SD of three independent experiments. (B) Phosphorylation of p38 MAPK was observed by immunoblot analysis in control NIH3T3 cells and 14-3-3ع-expressing cells at the indicated time. Phosphorylation of ERK and expression of MMP-2 protein were examined as controls. Bots shown in this experiment were typical of three independent experiments. 
stion, three double-stranded oligonucleotides, including a wild type14ERE sequence (14EREwt), mutations in the p53BS sequence (53BSmt) and mutations in the 14BS sequence (14BSmt), were cloned into pGL2-pro reporter plasmids, respectively (Supplemental Data Table S1). These reporter plasmids were co-transfected into NIH3T3 cells in the presence or absence of $14-3-3 \varepsilon$. The wild type 14ERE region and the 53BSmt region with mutations of the p53BS sequence were still activated by $14-3-3 \varepsilon$ protein, whereas the $14 \mathrm{BSmt}$ region with mutations of the 14BS sequence was not (Figure 3F). These promoter activities were consistent with the results from EMSA that 14-3-3e induced a specific DNA binding protein to bind to the 14BS sequence. Therefore, our results strongly suggest that the 14BS sequence from $-1,629$ to $-1,612$ of the MMP-2 promoter is an important cis-acting element for 14-3-3e-mediated MMP-2 gene expression.

We next examined the signal transduction pathway by which $14-3-3 \varepsilon$ stimulated MMP-2 gene expression. Interestingly, it has been reported that keratinocyte releasable stratifin, also known as $14-3-3 \sigma$, induced MMP-1 gene expression via p38 MAPK signaling (Lam et al., 2005). For this reason, we initially investigated the involvement of p38 MAPK signaling in 14-3-3e-mediated MMP-2 gene expression in $\mathrm{NIH} 3 \mathrm{~T} 3$ cells. A plasmid expressing a dominant negative p38 MAPK (DN-p38 MAPK) was co-transfected with the full-length MMP-2 promoter $(-1,659 /+57)$ into NIH3T3 cells in the presence or absence of 14-3-3e. As shown in Figure 4A, DN-p38 MAPK blocked the activation of the MMP-2 promoter by $14-3-3 \varepsilon$, whereas a plasmid of a dominant negative stress-activated protein kinase (DN-SEK) did not (Figure 4A). In addition, treatments of kinase inhibitors, wortmanin for phosphatidylinositide 3-kinase (PI3-kinase) and PD98059 for MEK1/2 kinase, did not affect 14-3- 38-mediated MMP-2 expression, but treatment of p38 MAPK inhibitor, SB203580, inhibited the activation of MMP-2 gene by $14-3-3 \varepsilon$ (data not shown). Furthermore, to clearly show that $14-3-3 \varepsilon$ stimulates p38 MAPK signaling, we examined the phosphorylation of p38 MAPK in 14-3-3e-expressing NIH3T3 cells. Compared with control cells expressing empty vector, phosphorylation of p38 MAPK was significantly increased in 14-3-3ع-expressing NIH3T3 cells, in which expression of MMP-2 protein was also elevated (Figure 4B). However, phosphorylation of ERK was not affected by $14-3-3 \varepsilon$ expression. Collectively, these results strongly demonstrate that expression of the $14-3-3 \varepsilon$ protein activates the MMP-2 promoter via p38 MAPK signaling.

\section{Discussion}

In this study, we demonstrated a novel mechanism leading to MMP-2 expression by $14-3-3 \varepsilon$ and a possible role for $14-3-3 \varepsilon$ that was overexpressed in aged skin. Our results indicate that $14-3-3 \varepsilon$ up-regulates MMP-2 mRNA at the transcriptional level by inducing a specific DNA binding protein bound to the upstream 14BS sequences $(-1,629$ to $-1,612)$ of the MMP-2 promoter. To our knowledge, this is the first report showing that $14-3-3 \varepsilon$ is able to increase the expression of the MMP-2 gene. In addition, the induction of MMP-2 mRNA by $14-3-3 \varepsilon$ was shown to be mediated by p38 MAPK signaling. And, the augmentation of MMP-2 mRNA by $14-3-3 \varepsilon$ was independent of the level of tissue inhibitor of matrix metalloprotease-2 (TIMP-2) (data not shown). These findings are supported, in part, by previous

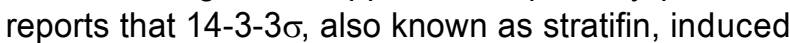
MMP-1 (Ghahary et al., 2004, 2005). Ghahary et al. (2005) reported that keratinocyte releasable 14-3-3 $\sigma$ stimulated MMP-1 mRNA and protein levels in dermal fibroblasts. Induction of MMP-1 mRNA and protein level by $14-3-3 \sigma$ was mediated via p38 MAPK signaling (Lam et al., 2005). Based on these previous reports and our present findings, it appears that expressions of 14-3-3 proteins in fibroblast cells are involved in ECM turnover by increasing MMP proteins.

The more interesting finding in this study is the upstream sequence of the MMP-2 promoter from $-1,629$ to $-1,612$, designated 14BS. This region was originally characterized as a silencer element in astroglioma U251-MG cells (Qin et al., 1999). However, deletion of this region in our study slightly decreased the activity of the MMP-2 promoter activity in the absence of 14-3-3e. Furthermore, analysis of deletion mutants of the MMP-2 promoter and EMSA apparently indicated that this region from $-1,629$ to $-1,612$ is required for activation of 14-3-3e-mediated MMP-2 promoter, and that a distinct DNA binding protein induced by $14-3-3 \varepsilon$ specifically binds to this region (Figure 3 ). These results suggest that the 14BS region identified in this study could not act as a silencer element in $\mathrm{NIH} 3 \mathrm{~T} 3$ fibroblast cells, and this discrepancy appears to be due to differences of biological contexts between those cell lines.

The disruption of the homeostatic balance between collagen synthesis and degradation leads to collagen deficiency in the dermis and eventually causes wrinkle formation (Fisher et al., 1997; Varani et al., 2000). We previously showed that $14-3-3 \varepsilon$ is overexpressed in naturally aged and photoaged human skin and its expression is induced by ultraviolet irradiation (Choi et al., 2005). Based on our 
present results, it is thought that overexpression of 14-3-3 $\varepsilon$ leads to wrinkle formation in aged skin by increasing MMP-2 mRNA and protein levels. That is, up-regulation of MMP-2 by overexpression of 14-3-3e presumably stimulates collagen deficiency through the degradation of type IV collagen, which subsequently contributes to wrinkle formation.

In conclusion, we demonstrate here a biological context for the overexpression of $14-3-3 \varepsilon$ in naturally aged and photoaged human skin, and reveal an underlying mechanism for $14-3-3 \varepsilon$ that increases the expression of the MMP-2 gene. Therefore, our results imply that modulation of $14-3-3 \varepsilon$ expression may be an important target for the control of wrinkle formation in aged skin.

\section{Methods}

\section{Plasmids}

To construct a recombinant retroviral plasmid expressing the $14-3-3 \varepsilon$ gene, the open reading frame of the $14-3-3 \varepsilon$ gene tagged with an hemagglutinin (HA) epitope was amplified by specific primers (Supplemental Data Table S1) and cloned into a pMSCV-puro retroviral vector (Clonetech), resulting in pMSCV-14-3-3e. For transient transfection, the 14-3-3ع gene was subcloned into a pSG5-HA vector, generating pSG5-HA-14-3-3 $\varepsilon$. The full-length MMP-2 promoter $(-1,659 /+57)$ was kindly provided by Dr. Seung Taek Lee (Yonsei University, Korea). Serial deletion mutants of the MMP-2 promoter $(-1,659 /+57,-1,611 /+57,-1,591 /+57$, $-1,259 /+57,-1,050 /+57$, and $-562 /+57$ ) were PCR-amplified and subcloned into a pGL3-basic vector as previously described (Qin et al, 1999). To identify which sequences of the MMP-2 promoter act as an important cis-acting element for 14-3-3ع-mediated MMP-2 expression, three double-stranded oligonucleotides were cloned into a pGL2-pro reporter plasmid, resulting in p14EREwt-Luc, p53BSmt-luc and p14BSmt-Luc, respectively. The oligonucleotide sequences of these reporter plasmids and their descriptions are shown in Supplemental Data Table S1. The PCR-generated and oligonucleotide sequences were verified by DNA sequencing. Plasmids of a dominantnegative p38 MAP kinase (DN-p38 MAPK) and a dominant negative stress-activated protein kinase (DN-SEK) were kindly provided by Dr. Aree Moon (Duksung Women's University, Korea) and Dr. Byung-Chul Kim (Kangwon National University, Korea), respectively.

\section{Cell lines, transient transfection, reporter assays, antibodies, and siRNA}

$\mathrm{NIH} 3 \mathrm{~T} 3$ fibroblast cells were maintained in DMEM supplemented with $10 \%$ FBS (GIBCO) at $37^{\circ} \mathrm{C}$ in a humidified environment containing $5 \% \mathrm{CO}_{2}$. For gelatin zymography and expressions of MMP-2 protein and mRNA, 14-3-3عexpressing NIH3T3 cells and control cells were cultured in serum-free DMEM for the indicated times. The production of recombinant retroviruses and generation of stable cells expressing the $14-3-3 \varepsilon$ gene were performed as previously described (Lee et al., 2007b). Cells in each of six-well plates were transiently transfected with the indicated plasmids using Effectene (Qiagen, Sweden) according to the manufacturer's protocol. Luciferase activities were normalized to $\beta$-galactosidase activity to adjust for variations in transfection efficiency. All experiments were independently repeated at least 3 times with similar results. Preparation of whole cell extracts and immunoblot analysis were performed as previously described (Lee et al., 2007a). For immunoblot, antibodies for mouse anti-HA (F-7), rabbit anti-14-3-3e (T-16), rabbit anti-MMP-2 (H-76), goat anti-MMP-9 (M-17), mouse-anti-p38 MAPK (A-12), rabbit-anti-ERK (K-23), and mouse-anti-phospho-ERK (E-4) were purchased from Santa Cruz Biotechnology. Anti-phospho-p38 MAPK and anti- $\beta$-actin antibodies were obtained from Cell signaling technology (Beverly, MA) and Sigma (St Louis, MO), respectively. To inhibit endogenous $14-3-3 \varepsilon$ gene expression, a synthetic siRNA duplex against $14-3-3 \varepsilon$ mRNA was purchased from Santa Cruz Biotechnology. $14-3-3 \varepsilon$ siRNA duplexes were transfected into NIH3T3 cells using Lipofectamine 2000 (Invitrogen) according to manufacturer's protocol.

\section{Gelatin zymography}

Conditioned media were collected at the indicated times and centrifuged at $3,000 \mathrm{rpm}$ for $10 \mathrm{~min}$ to remove cell debris. Gelatinolytic activities of the conditioned media were determined by gelatin zymogram assay as previously described (Shin et al., 2005).

\section{RNA extraction and RT-PCR}

Total RNA extraction and reverse transcription were done as previously described (Lee et al., 2007b). Specific primers for amplification of MMP-2, MMP-9 and 14-3-3e genes are shown in Supplemental Data Table S1. As an internal control, the GAPDH gene was amplified. PCR amplifications were done for 35 cycles consisting of denaturation at $94^{\circ} \mathrm{C}$ for $40 \mathrm{~s}$, annealing at $56^{\circ} \mathrm{C}$ for $60 \mathrm{~s}$, and extension at $72^{\circ} \mathrm{C}$ for $90 \mathrm{~s}$.

\section{Nuclear extracts and eletrophoretic mobility shift assay (EMSA)}

The preparation of nuclear extracts and EMSA were performed essentially as described (Park et al., 2001). For EMSA, double-stranded oligonucleotides of the 14ERE region were labeled with $\left[\gamma^{32} \mathrm{P}\right] \mathrm{ATP}$ and polynucleotide kinase. For competition assay, double-stranded oligonucleotides, designated p53BS and 14BS, were used as cold competitors. The sequences for 14ERE, p53BS and 14BS oligonucleotides were as follows: 14ERE, 5'-CACACCCACCAGACAAGCCTGAACTTGTCTGAAGCCCACTGAGAC CCA-3'; p53BS, 5'-AGACAAGCCTGAACTTGTCT-3'; 14BS, 5'-GAAGCCCACTGAGACCCA-3'. 


\section{Supplemental data}

Supplemental Data include a table and can be found with this article online at http://e-emm.or.kr/article/article_files/ SP-41-7-01.pdf.

\section{Acknowledgements}

This work was supported by grants from the Korea Health21 R\&D project, Ministry of Health and Welfare, Republic of Korea (A060471 to S.H.P) and by Korea Research Foundation Grants funded by the Korean Government (KRF-C00124 and KRF-E00016 to S.H.P and KRF-2005070-C00090 to C.Y.C).

\section{References}

Brinckerhoff CE, Matrisian LM. Matrix metalloproteinases: a tail of a frog that became a prince. Nat Rev Mol Cell Biol 2002;3;207-14

Choi KC, Lee S, Kwak SY, Kim MS, Choi HK, Kim KH, Chung $\mathrm{JH}$, Park SH. Increased expression of 14-3-3epsilon protein in intrinsically aged and photoaged human skin in vivo. Mech Ageing Dev 2005;126;629-36

Chung JH, Seo JY, Choi HR, Lee MK, Youn CS, Rhie G, Cho $\mathrm{KH}$, Kim KH, Park KC, Eun HC. Modulation of skin collagen metabolism in aged and photoaged human skin in vivo. J Invest Dermatol 2001;117;1218-24

Fisher GJ, Datta SC, Talwar HS, Wang ZQ, Varani J, Kang $\mathrm{S}$, Voorhees JJ. Molecular basis of sun-induced premature skin ageing and retinoid antagonism. Nature 1996;379: 335-9

Fisher GJ, Wang ZQ, Datta SC, Varani J, Kang S, Voorhees JJ. Pathophysiology of premature skin aging induced by ultraviolet light. N Engl J Med 1997;337:1419-28

Fu H, Subramanian RR, Masters SC. 14-3-3 proteins: structure, function, and regulation. Ann Rev Pharmacol Toxicol 2000;40:617-47

Ghahary A, Shen YJ, Nedelec B, Wang R, Scott PG, Tredget $E E$. Collagenase production is lower in post-burn hypertrophic scar fibroblasts than in normal fibroblasts and is reduced by insulin-like growth factor-1. J Invest Dermatol 1996;106:476-81

Ghahary A, Karimi-Busheri F, Marcoux Y, Li Y, Tredget EE, Taghi Kilani R, Li L, Zheng J, Karami A, Keller BO, Weinfeld M. Keratinocyte-releasable stratifin functions as a potent collagenase-stimulating factor in fibroblasts. J Invest Dermatol 2004;122:1188-97

Ghahary A, Marcoux Y, Karimi-Busheri F, Li Y, Tredget EE, Kilani RT, Lam E, Weinfeld M. Differentiated keratinocytereleasable stratifin (14-3-3 sigma) stimulates MMP-1 expression in dermal fibroblasts. J Invest Dermatol 2005;124: 170-7

Inomata S, Matsunaga Y, Amano S, Takada K, Kobayashi K, Tsunenaga M, Nishiyama T, Kohno Y, Fukuda M. Possible involvement of gelatinases in basement membrane damage and wrinkle formation in chronically ultraviolet B-exposed hairless mouse. J Invest Dermatol 2003;120:128-34

Kurz EU, Leader KB, Kroll DJ, Clark M, Gieseler F. Modulation of human DNA topoisomerase II alpha function by interaction with 14-3-3epsilon. J Biol Chem 2000;275: 13948-54

Lam E, Kilani RT, Li Y, Tredget EE, Ghahary A. Stratifininduced matrix metalloproteinase- 1 in fibroblast is mediated by $c$-fos and p38 mitogen-activated protein kinase activation. J Invest Dermatol 2005;125:230-8

Lee EJ, Choi EM, Kim SR, Park JH, Kim H, Ha KS, Kim YM, Kim SS, Choe M, Kim JI, Han JA. Cyclooxygenase-2 promotes cell proliferation, migration and invasion in U2OS human osteosarcoma cells. Exp Mol Med 2007a;39:469-76

Lee EK, Lee YS, Han IO, Park SH. Expression of Caveolin-1 reduces cellular responses to TGF-beta1 through downregulating the expression of TGF-beta type II receptor gene in NIH3T3 fibroblast cells. Biochem Biophys Res Commun 2007b;359:385-90

Nagase H, Woessner Jr JF. Matrix metalloproteinases. J Biol Chem 1999;274:21491-4

Park SH, Birchenall-Roberts MC, Yi Y, Lee BI, Lee DK, Bertolette DC, Fu T, Ruscetti F, Kim SJ. Mechanism of induction of transforming growth factor-beta type II receptor gene expression by v-Src in murine myeloid cells. Cell Growth Differ 2001;12:9-18

Qin H, Sun Y, Benveniste EN. The transcription factors Sp1, $\mathrm{Sp} 3$, and AP-2 are required for constitutive matrix metalloproteinase-2 gene expression in astroglioma cells. J Biol Chem 1999;274:29130-7

Rittie L, Fisher GJ. UV-light-induced signal cascades and skin aging. Ageing Res Rev 2002;1:705-20

Scharffetter K, Wlaschek M, Hogg A, Bolsen K, Schothorst A, Goerz G, Krieg T, Plewig G. UVA irradiation induces collagenase in human dermal fibroblasts in vitro and in vivo. Arch Dermatol Res 1991;283:506-11

Shin JY, Hur W, Wang JS, Jang JW, Kim CW, Bae SH, Jang SK, Yang SH, Sung YC, Kwon OJ, Yoon SK. HCV core protein promotes liver fibrogenesis via up-regulation of CTGF with TGF-beta1. Exp Mol Med 2005;37:138-45

Tak H, Jang E, S. Kim SB, Park J, Suk J, Yoon YS, Ahn JK, Lee JH, Joe CO. 14-3-3epsilon inhibits MK5-mediated cell migration by disrupting F-actin polymerization. Cell Signal 2007; 19:2379-87

Toyo-oka K, Shionoya A, Gambello MJ, Cardoso C, Leventer R, Ward HL, Ayala R, Tsai LH, Dobyns W, Ledbetter D, Hirotsune S, Wynshaw-Boris A. 14-3-3epsilon is important for neuronal migration by binding to NUDEL: a molecular explanation for Miller-Dieker syndrome. Nat Genet 2003;34: 274-85

van Hemert MJ, Steensma HY, van Heusden GP. 14-3-3 proteins: key regulators of cell division, signalling and apoptosis. Bioessays 2001;23:936-46

Varani J, Warner RL, Gharaee-Kermani M, Phan SH, Kang 
S, Chung JH, Wang ZQ, Datta SC, Fisher GJ, Voorhees JJ. Vitamin A antagonizes decreased cell growth and elevated collagen-degrading matrix metalloproteinases and stimulates collagen accumulation in naturally aged human skin. $J$ Invest Dermatol 2000;114:480-6
Visse R, Nagase H. Matrix metalloproteinases and tissue inhibitors of metalloproteinases: structure, function, and biochemistry. Circ Res 2003;92:827-39

Wilker E, Yaffe MB. 14-3-3 Proteins: a focus on cancer and human disease. J Mol Cell Cardiol 2004;37:633-42 